\title{
The changing face of Irish head and neck cancer epidemiology: 20 years of data
}

\author{
Gerard P. Sexton ${ }^{1,2}$ (D) Paul Walsh $^{3} \cdot$ Frank Moriarty $^{2,4} \cdot$ James Paul O'Neill $^{1,2}$
}

Received: 7 March 2021 / Accepted: 5 May 2021 / Published online: 13 October 2021

(c) The Author(s) 2021

\begin{abstract}
Background Head and neck cancer (HNC) is associated with significant morbidity and mortality, especially when high stage disease is present. The epidemiology and prognosis of HNC has changed considerably over the last 20 years.

Aims This study aimed to examine the epidemiological trends in HNC patients over a prolonged period in Ireland.

Methods We conducted a retrospective cohort study using 20 years of cancer registry data provided by the National Cancer Registry of Ireland. Baseline characteristics and survival statistics were thereby generated.

Results 10,148 patients were identified. There is a growing population of young ( $<50$ years) and very old ( $>85$ years) HNC patients; $48.15 \%$ of the population was elderly ( $>65$ years). Oral cavity $(29.8 \%)$ and laryngeal cancer $(28.1 \%)$ remain the most prevalent subsites, though oral cavity cancer prevalence declined from $35.9 \%$ in 1994 to $27.5 \%$ in 2014 . Oropharyngeal cancer prevalence increased from 13.6 to $22.2 \%$ over the same period. Overall 5-year survival has improved significantly to $56.8 \%$ in 2010 but there remains a disparity between the elderly and adult cohorts ( $42.0 \%$ vs $60.7 \%)$. 5-year survival for hypopharyngeal and oropharyngeal cancers has improved from $11.8 \%$ and $33.3 \%$ to $22.2 \%$ and $44.8 \%$, respectively, while laryngeal and oral cavity cancer survival remains approximately stable at $58.7 \%$ and $61.5 \%$, respectively.

Conclusion HNC survival in Ireland has improved in line with increasing recognition of the value of multidisciplinary assessment, subspecialisation in cancer care, and targeted therapies based on tumour subsites. Survival in the elderly cohort remains poor despite increasing recognition of the challenges such cases pose.
\end{abstract}

Keywords Head and neck surgery $\cdot$ Epidemiology $\cdot$ Laryngeal cancer $\cdot$ Oral cavity cancer $\cdot$ Nasal cavity cancer · Nasopharyngeal cancer · Oropharyngeal cancer · Hypopharyngeal cancer · Cancer survival · Elderly head and neck cancer

\section{Introduction}

Head and neck cancer (HNC) is a heterogeneous group of malignancies comprised of cancers of the oral cavity, oropharynx, nasopharynx, hypopharynx, larynx, salivary glands, nasal cavity, and paranasal sinuses [1]. HNC accounts for approximately $6 \%$ of new cancer diagnoses

Gerard P. Sexton

gerardsexton@rcsi.ie

1 Department of Otolaryngology-Head and Neck Surgery, Beaumont Hospital, Beaumont Road, Dublin 9, Ireland

2 Royal College of Surgeons in Ireland, Dublin, Ireland

3 National Cancer Registry Ireland, Cork Airport Business Park, Cork, Ireland

4 School of Pharmacy and Biomolecular Sciences, Royal College of Surgeons in Ireland, Dublin 2, Ireland worldwide [2]. Approximately a quarter of HNC occurs in elderly patients [3] with age and comorbidity often highlighted as prognostic predictors due to their effect on therapeutic tolerance [4]. There is also a significant association between HNC and socioeconomic deprivation [5]. The primary histopathological diagnosis is Squamous Cell Carcinoma (SCC), comprising well over $90 \%$ of cases [6], with a heavy preponderance of this patient group towards prolonged exposure to tobacco, alcohol, or both [7]. Human Papilloma Virus (HPV) infection has been increasingly recognised over the last 30 years as a major risk factor for the development of HNC, with oropharyngeal malignancy displaying such a strong association that HPV status now forms part of the staging process for such patients [1, 8-11]. There is a further association between nasopharyngeal malignancy and Epstein-Barr Virus (EBV) [12, 13]. Up to 70\% of HNC presents with either locally advanced or metastatic disease in the first instance [14]. There has up to this point been 
no aggregated analysis of the epidemiological data of Irish HNC patients.

\section{Aims and objectives}

This study aimed to examine the epidemiological trends in $\mathrm{HNC}$ patients over a prolonged period in Ireland. Specific objectives included determining the overall survival by stage, age, year of incidence, and location.

\section{Materials and methods}

\section{Study design}

A retrospective cohort study was conducted using STROBE standardised reporting guidelines. The study cohort was derived from a database obtained from the National Cancer Registry of Ireland (NCRI) of HNC patients diagnosed in Ireland between 1994 and 2014. The length of cancerspecific follow-up in this instance is until the end of 2015. This database was derived from both electronic healthcare records and physical charts which the NCRI analyses on a continual basis. The elderly population was predefined as anyone over 65 years of age. This age was chosen as the most often quoted definition of elderly in published literature both in $\mathrm{HNC}$ and in general [4].

\section{Inclusion/exclusion criteria}

The sole inclusion criterion was patients with HNC (as defined by TNM Classification of Malignant Tumours (TNM) for Head and Neck Cancer 8e [1]) diagnosed within the period specified. The primary exclusion criterion was patients with cancers occurring outside this region. Cutaneous and thyroid malignancies were also excluded owing to the considerable differences they display in terms of treatment and prognosis.

\section{Statistical methods}

Descriptive statistics for included participants' baseline characteristics were generated. Year of incidence data by site and age, overall stage at presentation statistics, and 5-year survival statistics by stage, year of incidence and site were also generated using Stata version 16.1.

\section{Ethical considerations}

Ethical approval was sought from and approved by the RCSI Research Ethics Committee. The database in question already exists, and the NCRI retains legislative authority to analyse data for research, and release data to external parties specifically for this purpose [15]. All data derived from the NCRI database are fully anonymised in line with best practice as outlined by the Data Protection Commission, and their lawful grounds for processing same is pursuant to compliance with a legal obligation [15]. Informed consent has not been explicitly sought from any of the patients involved.

\section{Results}

\section{Baseline characteristics}

Between January 1994 and December 2014, there were 10,148 HNC patients in the Republic of Ireland. The baseline characteristics of these patients are summarised in Table 1.

Elderly patients accounted for $48.1 \%(n=4886)$ of cases. The incidence of HNC in Ireland remained stable for the first 10 years of the study period but has subsequently risen year on year, with 434 cases in 1994 compared to 643 cases in $2014.74 .2 \%(n=7525)$ of patients were male. The predominant histopathological diagnosis was SCC in $84.3 \%$ $(n=8555)$, though there was a notable proportion of malignancies coded as "unspecified carcinoma" $(n=825,8.13 \%)$. Adenocarcinoma was the next most commonly occurring diagnosis in $6.2 \%(n=631)$.

There were 18 sites and 79 subsites reported in the dataset. These were analysed and grouped according to anatomical definitions laid out by the AJCC [16] into the seven specific groups detailed in Table 1. Lesions with sites that overlapped these groups or which were without a definite anatomical location were designated "other" $(n=366,3.6 \%)$. Oral cavity cancer was the most common malignancy in $29.8 \%(n=3028)$ followed closely by laryngeal cancer in $28.1 \%(n=2848)$ and oropharyngeal in $15.8 \%(1606)$. When broken down by gender, this pattern was replicated in female patients $(36.5 \%, 16.9 \%, 14.6 \%)$. In male patients, the larynx was the most commonly affected site $(32.2 \%, n=2423)$, followed by the oral cavity $(27 \%, n=2032)$.

Year of incidence data organised by disease site is presented in Table 2. The incidence of HNC rose across all disease sites, with the oral cavity, larynx, and oropharynx consistently the most prevalent. There was a decrease in the relative prevalence of oral cavity cancer from $35.9 \%$ in 1994 to $27.5 \%$ in 2014 . There was also an increase in the relative prevalence of oropharyngeal cancer from $13.6 \%$ in 1994 to $22.2 \%$ in 2014.

Year of incidence data organised by age group is presented in Table 3. The proportion of patients over 65 years changed over the 20 years observed-this group comprised $56.4 \%$ of cases in 1994 and $46.8 \%$ in 2014. Despite this there was a steady rise in the prevalence of $\mathrm{HNC}$ in patients over 
Table 1 Baseline characteristics of Irish head \& neck cancer patients 1994-2014

\begin{tabular}{|c|c|c|c|}
\hline Variable & Frequency & Percentage & Cumulative $\%$ \\
\hline \multicolumn{4}{|l|}{ Age group } \\
\hline 00_04 & 11 & 0.11 & 0.11 \\
\hline 05_09 & 12 & 0.12 & 0.23 \\
\hline 10_14 & 23 & 0.23 & 0.45 \\
\hline 15_19 & 33 & 0.33 & 0.78 \\
\hline 20_24 & 30 & 0.3 & 1.07 \\
\hline $25 \_29$ & 46 & 0.45 & 1.53 \\
\hline 30_34 & 106 & 1.04 & 2.57 \\
\hline 35_39 & 161 & 1.59 & 4.16 \\
\hline 40_44 & 297 & 2.93 & 7.09 \\
\hline 45_49 & 649 & 6.4 & 13.48 \\
\hline 50_54 & 990 & 9.76 & 23.24 \\
\hline 55_59 & 1,375 & 13.55 & 36.79 \\
\hline 60_64 & 1,529 & 15.07 & 51.85 \\
\hline 65_69 & 1,441 & 14.2 & 66.05 \\
\hline 70_74 & 1,270 & 12.51 & 78.57 \\
\hline 75_79 & 997 & 9.82 & 88.39 \\
\hline 80_84 & 674 & 6.64 & 95.03 \\
\hline $85+$ & 504 & 4.97 & 100 \\
\hline \multicolumn{4}{|l|}{ Year of incidence } \\
\hline 1994 & 434 & 4.28 & 4.28 \\
\hline 1995 & 431 & 4.25 & 8.52 \\
\hline 1996 & 417 & 4.11 & 12.63 \\
\hline 1997 & 405 & 3.99 & 16.62 \\
\hline 1998 & 427 & 4.21 & 20.83 \\
\hline 1999 & 397 & 3.91 & 24.74 \\
\hline 2000 & 414 & 4.08 & 28.82 \\
\hline 2001 & 319 & 3.14 & 31.97 \\
\hline 2002 & 440 & 4.34 & 36.3 \\
\hline 2003 & 422 & 4.16 & 40.46 \\
\hline 2004 & 452 & 4.45 & 44.92 \\
\hline 2005 & 468 & 4.61 & 49.53 \\
\hline 2006 & 482 & 4.75 & 54.28 \\
\hline 2007 & 489 & 4.82 & 59.1 \\
\hline 2008 & 504 & 4.97 & 64.06 \\
\hline 2009 & 536 & 5.28 & 69.34 \\
\hline 2010 & 603 & 5.94 & 75.29 \\
\hline 2011 & 603 & 5.94 & 81.23 \\
\hline 2012 & 601 & 5.92 & 87.15 \\
\hline 2013 & 661 & 6.51 & 93.66 \\
\hline 2014 & 643 & 6.34 & 100 \\
\hline \multicolumn{4}{|l|}{ Gender } \\
\hline $\mathrm{F}$ & 2,623 & 25.85 & 25.85 \\
\hline M & 7,525 & 74.15 & 100 \\
\hline \multicolumn{4}{|l|}{ Histology } \\
\hline Adenocarcinoma & 631 & 6.22 & 6.22 \\
\hline Melanoma & 61 & 0.6 & 6.82 \\
\hline Unspecified & 825 & 8.13 & 14.95 \\
\hline Sarcoma & 76 & 0.75 & 15.7 \\
\hline
\end{tabular}

Table 1 (continued)

\begin{tabular}{lccl}
\hline Variable & Frequency & Percentage & Cumulative \% \\
\hline Squamous cell carcinoma & 8,555 & 84.3 & 100 \\
Site & & & \\
Hypopharynx & 793 & 7.81 & 7.81 \\
Larynx & 2,848 & 28.06 & 35.88 \\
Nasal cavity/paranasal & 482 & 4.75 & 40.63 \\
$\quad$ sinuses & & & \\
Nasopharynx & 305 & 3.01 & 43.63 \\
Oral cavity & 3,028 & 29.84 & 73.47 \\
Oropharynx & 1,606 & 15.83 & 89.3 \\
Other & 366 & 3.61 & 92.91 \\
Salivary gland & 720 & 7.09 & 100 \\
\hline
\end{tabular}

85 years (3.9\% in 1994 to $5.3 \%$ in 2014). The prevalence of HNC in patients over 50 years and less than 65 years increased over the 20 years observed from $33.4 \%$ in 1994 to $38.1 \%$ in 2014 . A similar increase was seen in patients less than 50 years (10\% in 1994 vs. $15.2 \%$ in 2014).

\section{Stages and survival}

Stage at presentation statistics overall and organised by site are summarised in Table 4. Patients presenting with stage 0 , stage I or stage II disease comprised $30.3 \%(n=3075)$. Stage III disease was present in $10.9 \%(n=1102)$, non-metastatic stage IV disease was present in $24.3 \%(n=2469)$, and metastatic stage IV (stage IVC) disease was present in $3.9 \%$ (393). Oropharyngeal and hypopharyngeal cancers in particular presented with advanced disease in at least $63.3 \%$ and $44.4 \%$ of cases, respectively.

5-year survival statistics by stage and age group (divided into the adult and elderly populations) are summarised in Fig. 1. The overall 5-year survival for HNC from 16 years of Irish data was 52.2\%. 5-year survival from locoregionally confined disease (i.e. stage I and II) was $74.3 \%$. The best prognosis was conferred by stage I disease, with $82.5 \%$ of patients surviving to 5 years. Stage IVC disease conferred the worst prognosis, with $46.5 \%$ alive at 1 -year and $11.8 \%$ alive at 5 years.

A similar proportion of patients in both elderly and adult cohorts presented with each stage of disease. Survival was lower in the elderly cohort for all stages of disease. Formal record of disease stage was absent in 30.6\% $(n=3109)$ of cases (represented as stage $\mathrm{X}$ ). Following exclusion of cases which occurred in $2014,55.2 \%(n=1362)$ of cases where disease stage was not available occurred in elderly patients. The overall survival in this group, as shown in Fig. 1, is most analogous to stage III disease.

5 -year survival by stage and year of incidence is presented in Table 5. Overall 5-year survival improved over 
Table 2 Year of incidence head and neck cancer data organised by site

\begin{tabular}{|c|c|c|c|c|c|c|c|c|c|}
\hline \multicolumn{10}{|l|}{ Key } \\
\hline \multicolumn{10}{|c|}{ Frequency } \\
\hline \multicolumn{10}{|c|}{ Row percentage } \\
\hline Year & Hypopharynx & Larynx & $\begin{array}{l}\text { Nasal Cavity I } \\
\text { Paranasal Sinuses }\end{array}$ & Nasopharynx & Oral Cavity & Oropharynx & Other & $\begin{array}{l}\text { Salivary } \\
\text { Gland }\end{array}$ & Total \\
\hline \multirow[t]{2}{*}{1994} & 37 & 112 & 17 & 10 & 156 & 59 & 11 & 32 & 434 \\
\hline & $8.53 \%$ & $25.81 \%$ & $3.92 \%$ & $2.3 \%$ & $35.94 \%$ & $13.59 \%$ & $2.53 \%$ & $7.37 \%$ & $100 \%$ \\
\hline \multirow[t]{2}{*}{1995} & 33 & 118 & 19 & 13 & 142 & 59 & 17 & 30 & 431 \\
\hline & $7.66 \%$ & $27.38 \%$ & $4.41 \%$ & $3.02 \%$ & $32.95 \%$ & $13.69 \%$ & $3.94 \%$ & $6.96 \%$ & $100 \%$ \\
\hline \multirow{2}{*}{1996} & 53 & 106 & 17 & 17 & 135 & 42 & 19 & 28 & 417 \\
\hline & $12.71 \%$ & $25.42 \%$ & $4.08 \%$ & $4.08 \%$ & $32.37 \%$ & $10.07 \%$ & $4.56 \%$ & $6.71 \%$ & $100 \%$ \\
\hline \multirow{2}{*}{1997} & & & & & 123 & 54 & 18 & 34 & 405 \\
\hline & $\begin{array}{r}36 \\
8.89 \%\end{array}$ & $\begin{array}{r}102 \\
25.19 \%\end{array}$ & $\begin{array}{r}25 \\
6.17 \%\end{array}$ & $\begin{array}{r}13 \\
3.21 \%\end{array}$ & $\begin{array}{r}123 \\
30.37 \%\end{array}$ & $\begin{array}{r}54 \\
13.33 \%\end{array}$ & $\begin{array}{r}18 \\
4.44 \%\end{array}$ & $\begin{array}{r}34 \\
8.4 \%\end{array}$ & $\begin{array}{r}405 \\
100 \%\end{array}$ \\
\hline \multirow[t]{2}{*}{1998} & 30 & 116 & 28 & 10 & 150 & 42 & 19 & 32 & 427 \\
\hline & $7.03 \%$ & $27.17 \%$ & $6.56 \%$ & $2.34 \%$ & $35.13 \%$ & $9.84 \%$ & $4.45 \%$ & $7.49 \%$ & $100 \%$ \\
\hline \multirow{3}{*}{1999} & & & & & & & & & \\
\hline & 30 & 116 & 21 & 19 & 114 & 42 & 26 & 29 & 397 \\
\hline & $7.56 \%$ & $29.22 \%$ & $5.29 \%$ & $4.79 \%$ & $28.72 \%$ & $10.58 \%$ & $6.55 \%$ & $7.3 \%$ & $100 \%$ \\
\hline \multirow[t]{2}{*}{2000} & 36 & 132 & 21 & 12 & 120 & 53 & 18 & 22 & 414 \\
\hline & $8.7 \%$ & $31.88 \%$ & $5.07 \%$ & $2.9 \%$ & $28.99 \%$ & $12.8 \%$ & $4.35 \%$ & $5.31 \%$ & $100 \%$ \\
\hline \multirow[t]{2}{*}{2001} & 16 & 91 & 17 & 13 & 102 & 42 & 16 & 22 & 319 \\
\hline & $5.02 \%$ & $28.53 \%$ & $5.33 \%$ & $4.08 \%$ & $31.97 \%$ & $13.17 \%$ & $5.02 \%$ & $6.9 \%$ & $100 \%$ \\
\hline \multirow{2}{*}{2002} & 22 & 148 & 23 & 10 & 112 & 67 & 14 & 44 & 440 \\
\hline & $5 \%$ & $33.64 \%$ & $5.23 \%$ & $2.27 \%$ & $25.45 \%$ & $15.23 \%$ & $3.18 \%$ & $10 \%$ & $100 \%$ \\
\hline \multirow[t]{2}{*}{2003} & 36 & 133 & 22 & 12 & 113 & 62 & 13 & 31 & 422 \\
\hline & $8.53 \%$ & $31.52 \%$ & $5.21 \%$ & $2.84 \%$ & $26.78 \%$ & $14.69 \%$ & $3.08 \%$ & $7.35 \%$ & $100 \%$ \\
\hline \multirow[t]{2}{*}{2004} & 40 & 135 & 25 & 15 & 113 & 74 & 15 & 35 & 452 \\
\hline & $8.85 \%$ & $29.87 \%$ & $5.53 \%$ & $3.32 \%$ & $25 \%$ & $16.37 \%$ & $3.32 \%$ & $7.74 \%$ & $100 \%$ \\
\hline & & & & & & & & & \\
\hline \multirow[t]{2}{*}{2005} & 36 & 140 & 23 & 13 & 138 & 72 & 14 & 32 & 468 \\
\hline & $7.69 \%$ & $29.91 \%$ & $4.91 \%$ & $2.78 \%$ & $29.49 \%$ & $15.38 \%$ & $2.99 \%$ & $6.84 \%$ & $100 \%$ \\
\hline 2006 & 41 & 139 & 21 & 14 & 133 & 86 & 18 & 30 & 482 \\
\hline & $8.51 \%$ & $28.84 \%$ & $4.36 \%$ & $2.9 \%$ & $27.59 \%$ & $17.84 \%$ & $3.73 \%$ & $6.22 \%$ & $100 \%$ \\
\hline 2007 & 36 & 148 & 20 & 13 & 145 & 67 & 23 & 37 & 489 \\
\hline & $7.36 \%$ & $30.27 \%$ & $4.09 \%$ & $2.66 \%$ & $29.65 \%$ & $13.7 \%$ & $4.7 \%$ & $7.57 \%$ & $100 \%$ \\
\hline & & & & & & & & & \\
\hline 2008 & 35 & 152 & 25 & 16 & 146 & 75 & 15 & 40 & 504 \\
\hline & $6.94 \%$ & $30.16 \%$ & $4.96 \%$ & $3.17 \%$ & $28.97 \%$ & $14.88 \%$ & $2.98 \%$ & $7.94 \%$ & $100 \%$ \\
\hline 2009 & 52 & 135 & 22 & 14 & 158 & 95 & 20 & 40 & 536 \\
\hline & $9.7 \%$ & $25.19 \%$ & $4.1 \%$ & $2.61 \%$ & $29.48 \%$ & $17.72 \%$ & $3.73 \%$ & $7.46 \%$ & $100 \%$ \\
\hline & & & & & & & & & \\
\hline 2010 & 33 & 170 & 31 & 24 & 183 & 103 & 21 & 38 & 603 \\
\hline & $5.47 \%$ & $28.19 \%$ & $5.14 \%$ & $3.98 \%$ & $30.35 \%$ & $17.08 \%$ & $3.48 \%$ & $6.3 \%$ & $100 \%$ \\
\hline 2011 & 41 & 161 & 19 & 18 & 183 & 127 & 14 & 40 & 603 \\
\hline & $6.8 \%$ & $26.7 \%$ & $3.15 \%$ & $2.99 \%$ & $30.35 \%$ & $21.06 \%$ & $2.32 \%$ & $6.63 \%$ & $100 \%$ \\
\hline 2012 & & & & & & & & & \\
\hline 2012 & $\begin{array}{r}52 \\
865 \%\end{array}$ & $\begin{array}{r}167 \\
2779 \%\end{array}$ & $\begin{array}{r}27 \\
449 \%\end{array}$ & 18 & $\begin{array}{r}164 \\
2729 \%\end{array}$ & $\begin{array}{r}116 \\
193 \%\end{array}$ & $\begin{array}{r}24 \\
309 \%\end{array}$ & $\begin{array}{r}33 \\
549 \%\end{array}$ & 601 \\
\hline & $8.65 \%$ & $27.79 \%$ & $4.49 \%$ & $3 \%$ & $27.29 \%$ & $19.3 \%$ & $3.99 \%$ & $5.49 \%$ & $100 \%$ \\
\hline 2013 & 42 & 174 & 22 & 16 & 221 & 126 & 20 & 40 & 661 \\
\hline & $6.35 \%$ & $26.32 \%$ & $3.33 \%$ & $2.42 \%$ & $33.43 \%$ & $19.06 \%$ & $3.03 \%$ & $6.05 \%$ & $100 \%$ \\
\hline 2014 & 56 & 153 & 37 & 15 & 177 & 143 & 11 & 51 & 643 \\
\hline & $8.71 \%$ & $23.79 \%$ & $5.75 \%$ & $2.33 \%$ & $27.53 \%$ & $22.24 \%$ & $1.71 \%$ & $7.93 \%$ & $100 \%$ \\
\hline Total & 793 & 2,848 & 482 & 305 & 3,028 & 1,606 & 366 & 720 & 10,148 \\
\hline & $7.81 \%$ & $28.06 \%$ & $4.75 \%$ & $3.01 \%$ & $29.84 \%$ & $15.83 \%$ & $3.61 \%$ & $7.09 \%$ & $100 \%$ \\
\hline
\end{tabular}

the study period, with $46.5 \%$ of patients in 1994 surviving to 5 years compared to $56.8 \%$ in 2010 . All stages of disease showed improvements in survivability including stage III disease, from $45.3 \%$ in 1994 to $58.2 \%$ in 2010, and stage IV disease, from $24.1 \%$ in 1994 to $38.4 \%$ in 2010 .
Survival statistics by site and by year of incidence are detailed in Table 6. Overall survival was highest in oral cavity cancer ( $58.9 \%$ alive at 5 years) closely followed by salivary gland and laryngeal cancer $(58.6 \%$ and $58.6 \%$ alive at 5 years, respectively) and was worst with hypopharyngeal 
Table 3 Year of incidence head and neck cancer data organised by age group

\begin{tabular}{|c|c|c|c|c|c|c|c|c|c|c|c|c|c|c|c|c|c|c|c|}
\hline $\begin{array}{l}\text { Key } \\
\text { Frequ } \\
\text { Row }\end{array}$ & $\begin{array}{l}\text { ency } \\
\text { ercenta }\end{array}$ & & & & & & & & & & & & & & & & & & \\
\hline Yol & 00_04 & 05_09 & 10_14 & 15_19 & 20_24 & 25_29 & 30_34 & 35_39 & 40_44 & 45_49 & 50_54 & 55_59 & 60_64 & 65 69 & 70_74 & 75_79 & 80_84 & $85+$ & Total \\
\hline \multirow[t]{2}{*}{1994} & 1 & 1 & 1 & 0 & 1 & 0 & 2 & 4 & 10 & 24 & 35 & 44 & 66 & 71 & 73 & 46 & 38 & 17 & 434 \\
\hline & $0.2 \%$ & $0.2 \%$ & $0.2 \%$ & $0.0 \%$ & $0.2 \%$ & $0.0 \%$ & $0.5 \%$ & $0.9 \%$ & $2.3 \%$ & $5.5 \%$ & $8.1 \%$ & $10.1 \%$ & $15.2 \%$ & $16.4 \%$ & $16.8 \%$ & $10.6 \%$ & $8.8 \%$ & $3.9 \%$ & $100.0 \%$ \\
\hline \multirow[t]{2}{*}{1995} & 0 & 1 & 2 & 0 & 1 & 2 & 2 & 7 & 12 & 22 & 41 & 56 & 47 & 50 & 73 & 54 & 44 & 17 & 431 \\
\hline & $0.0 \%$ & $0.2 \%$ & $0.5 \%$ & $0.0 \%$ & $0.2 \%$ & $0.5 \%$ & $0.5 \%$ & $1.6 \%$ & $2.8 \%$ & $5.1 \%$ & $9.5 \%$ & $13.0 \%$ & $10.9 \%$ & $11.6 \%$ & $16.9 \%$ & $12.5 \%$ & $10.2 \%$ & $3.9 \%$ & $100.0 \%$ \\
\hline \multirow[t]{2}{*}{1996} & 0 & 1 & 1 & 0 & 1 & 2 & 5 & 5 & 8 & 33 & 31 & 49 & 58 & 68 & 49 & 56 & 33 & 17 & 417 \\
\hline & $0.0 \%$ & $0.2 \%$ & $0.2 \%$ & $0.0 \%$ & $0.2 \%$ & $0.5 \%$ & $1.2 \%$ & $1.2 \%$ & $1.9 \%$ & $7.9 \%$ & $7.4 \%$ & $11.8 \%$ & $13.9 \%$ & $16.3 \%$ & $11.8 \%$ & $13.4 \%$ & $7.9 \%$ & $4.1 \%$ & $100.0 \%$ \\
\hline \multirow[t]{2}{*}{1997} & 1 & 1 & 1 & 1 & 1 & 0 & 4 & 5 & 7 & 27 & 34 & 46 & 67 & 55 & 58 & 46 & 27 & 24 & 405 \\
\hline & $0.2 \%$ & $0.2 \%$ & $0.2 \%$ & $0.2 \%$ & $0.2 \%$ & $0.0 \%$ & $1.0 \%$ & $1.2 \%$ & $1.7 \%$ & $6.7 \%$ & $8.4 \%$ & $11.4 \%$ & $16.5 \%$ & $13.6 \%$ & $14.3 \%$ & $11.4 \%$ & $6.7 \%$ & $5.9 \%$ & $100.0 \%$ \\
\hline \multirow[t]{2}{*}{1998} & 0 & 0 & 2 & 2 & 0 & 0 & 4 & 6 & 13 & 33 & 38 & 46 & 61 & 58 & 54 & 53 & 38 & 19 & 427 \\
\hline & $0.0 \%$ & $0.0 \%$ & $0.5 \%$ & $0.5 \%$ & $0.0 \%$ & $0.0 \%$ & $0.9 \%$ & $1.4 \%$ & $3.0 \%$ & $7.7 \%$ & $8.9 \%$ & $10.8 \%$ & $14.3 \%$ & $13.6 \%$ & $12.6 \%$ & $12.4 \%$ & $8.9 \%$ & $4.4 \%$ & $100.0 \%$ \\
\hline \multirow[t]{2}{*}{1999} & 1 & 0 & 0 & 3 & 1 & 4 & 4 & 4 & 12 & 14 & 31 & 58 & 60 & 55 & 48 & 52 & 35 & 15 & 397 \\
\hline & $0.3 \%$ & $0.0 \%$ & $0.0 \%$ & $0.8 \%$ & $0.3 \%$ & $1.0 \%$ & $1.0 \%$ & $1.0 \%$ & $3.0 \%$ & $3.5 \%$ & $7.8 \%$ & $14.6 \%$ & $15.1 \%$ & $13.9 \%$ & $12.1 \%$ & $13.1 \%$ & $8.8 \%$ & $3.8 \%$ & $100.0 \%$ \\
\hline \multirow[t]{2}{*}{2000} & 1 & 1 & 3 & 1 & 3 & 2 & 5 & 6 & 11 & 29 & 44 & 57 & 55 & 56 & 43 & 35 & 38 & 24 & 414 \\
\hline & $0.2 \%$ & $0.2 \%$ & $0.7 \%$ & $0.2 \%$ & $0.7 \%$ & $0.5 \%$ & $1.2 \%$ & $1.4 \%$ & $2.7 \%$ & $7.0 \%$ & $10.6 \%$ & $13.8 \%$ & $13.3 \%$ & $13.5 \%$ & $10.4 \%$ & $8.5 \%$ & $9.2 \%$ & $5.8 \%$ & $100.0 \%$ \\
\hline \multirow[t]{2}{*}{2001} & 0 & 1 & 0 & 0 & 0 & 2 & 3 & 3 & 8 & 21 & 46 & 48 & 46 & 41 & 48 & 22 & 15 & 15 & 319 \\
\hline & $0.0 \%$ & $0.3 \%$ & $0.0 \%$ & $0.0 \%$ & $0.0 \%$ & $0.6 \%$ & $0.9 \%$ & $0.9 \%$ & $2.5 \%$ & $6.6 \%$ & $14.4 \%$ & $15.0 \%$ & $14.4 \%$ & $12.9 \%$ & $15.0 \%$ & $6.9 \%$ & $4.7 \%$ & $4.7 \%$ & $100.0 \%$ \\
\hline \multirow[t]{2}{*}{2002} & 0 & 2 & 0 & 2 & 2 & 1 & 3 & 8 & 12 & 26 & 42 & 67 & 58 & 43 & 64 & 56 & 29 & 25 & 440 \\
\hline & $0.0 \%$ & $0.5 \%$ & $0.0 \%$ & $0.5 \%$ & $0.5 \%$ & $0.2 \%$ & $0.7 \%$ & $1.8 \%$ & $2.7 \%$ & $5.9 \%$ & $9.5 \%$ & $15.2 \%$ & $13.2 \%$ & $9.8 \%$ & $14.5 \%$ & $12.7 \%$ & $6.6 \%$ & $5.7 \%$ & $100.0 \%$ \\
\hline \multirow[t]{2}{*}{2003} & 0 & 0 & 0 & 2 & 1 & 1 & 8 & 6 & 15 & 28 & 45 & 60 & 52 & 52 & 60 & 44 & 29 & 19 & 422 \\
\hline & $0.0 \%$ & $0.0 \%$ & $0.0 \%$ & $0.5 \%$ & $0.2 \%$ & $0.2 \%$ & $1.9 \%$ & $1.4 \%$ & $3.6 \%$ & $6.6 \%$ & $10.7 \%$ & $14.2 \%$ & $12.3 \%$ & $12.3 \%$ & $14.2 \%$ & $10.4 \%$ & $6.9 \%$ & $4.5 \%$ & $100.0 \%$ \\
\hline \multirow[t]{2}{*}{2004} & 0 & 0 & 1 & 1 & 0 & 0 & 6 & 8 & 12 & 15 & 54 & 69 & 55 & 63 & 64 & 38 & 37 & 29 & 452 \\
\hline & $0.0 \%$ & $0.0 \%$ & $0.2 \%$ & $0.2 \%$ & $0.0 \%$ & $0.0 \%$ & $1.3 \%$ & $1.8 \%$ & $2.7 \%$ & $3.3 \%$ & $11.9 \%$ & $15.3 \%$ & $2.2 \%$ & $13.9 \%$ & $14.2 \%$ & $8.4 \%$ & $8.2 \%$ & $6.4 \%$ & $100.0 \%$ \\
\hline \multirow[t]{2}{*}{2005} & 1 & 0 & 0 & 4 & 1 & 5 & 3 & 10 & 11 & 43 & 42 & 61 & 77 & 66 & 50 & 42 & 28 & 24 & 468 \\
\hline & $0.2 \%$ & $0.0 \%$ & $0.0 \%$ & $0.9 \%$ & $0.2 \%$ & $1.1 \%$ & $0.6 \%$ & $2.1 \%$ & $2.4 \%$ & $9.2 \%$ & $9.0 \%$ & $13.0 \%$ & $6.5 \%$ & $14.1 \%$ & $10.7 \%$ & $9.0 \%$ & $6.0 \%$ & $5.1 \%$ & $100.0 \%$ \\
\hline \multirow[t]{2}{*}{2006} & 1 & 0 & 0 & 1 & 2 & 2 & 3 & 8 & 15 & 35 & 58 & 67 & 74 & 73 & 58 & 34 & 24 & 27 & 482 \\
\hline & $0.2 \%$ & $0.0 \%$ & $0.0 \%$ & $0.2 \%$ & $0.4 \%$ & $0.4 \%$ & $0.6 \%$ & $1.7 \%$ & $3.1 \%$ & $7.3 \%$ & $12.0 \%$ & $13.9 \%$ & $15.4 \%$ & $15.1 \%$ & $12.0 \%$ & $7.1 \%$ & $5.0 \%$ & $5.6 \%$ & $100.0 \%$ \\
\hline \multirow[t]{2}{*}{2007} & 0 & 0 & 1 & 5 & 1 & 3 & 9 & 11 & 15 & 34 & 42 & 73 & 63 & 80 & 45 & 45 & 33 & 29 & 489 \\
\hline & $0.0 \%$ & $0.0 \%$ & $0.2 \%$ & $1.0 \%$ & $0.2 \%$ & $0.6 \%$ & $1.8 \%$ & $2.2 \%$ & $3.1 \%$ & $7.0 \%$ & $8.6 \%$ & $14.9 \%$ & $12.9 \%$ & $16.4 \%$ & $9.2 \%$ & $9.2 \%$ & $6.7 \%$ & $5.9 \%$ & $100.0 \%$ \\
\hline 2008 & 0 & 0 & 1 & 3 & 3 & 2 & 6 & 5 & 15 & 30 & 46 & 62 & 88 & 70 & 62 & 57 & 29 & 25 & 504 \\
\hline & $0.0 \%$ & $0.0 \%$ & $0.2 \%$ & $0.6 \%$ & $0.6 \%$ & $0.4 \%$ & $1.2 \%$ & $1.0 \%$ & $3.0 \%$ & $6.0 \%$ & $9.1 \%$ & $12.3 \%$ & $17.5 \%$ & $13.9 \%$ & $12.3 \%$ & $11.3 \%$ & $5.8 \%$ & $5.0 \%$ & $100.0 \%$ \\
\hline 2009 & 0 & 1 & 2 & 2 & 6 & 1 & 7 & 9 & 15 & 32 & 48 & 74 & 81 & 82 & 68 & 46 & 28 & 34 & 536 \\
\hline & $0.0 \%$ & $0.2 \%$ & $0.4 \%$ & $0.4 \%$ & $1.1 \%$ & $0.2 \%$ & $1.3 \%$ & $1.7 \%$ & $2.8 \%$ & $6.0 \%$ & $9.0 \%$ & $13.8 \%$ & $15.1 \%$ & $15.3 \%$ & $12.7 \%$ & $8.6 \%$ & $5.2 \%$ & $6.3 \%$ & $100.0 \%$ \\
\hline 2010 & 1 & 1 & 2 & 2 & 0 & 5 & 11 & 11 & 22 & 41 & 66 & 83 & 99 & 75 & 74 & 47 & 33 & 30 & 603 \\
\hline & $0.2 \%$ & $0.2 \%$ & $0.3 \%$ & $0.3 \%$ & $0.0 \%$ & $0.8 \%$ & $1.8 \%$ & $1.8 \%$ & $3.6 \%$ & $6.8 \%$ & $10.9 \%$ & $13.8 \%$ & $16.4 \%$ & $12.4 \%$ & $12.3 \%$ & $7.8 \%$ & $5.5 \%$ & $5.0 \%$ & $100.0 \%$ \\
\hline 2011 & 1 & 0 & 1 & 1 & 0 & 4 & 3 & 17 & 21 & 37 & 70 & 86 & 107 & 81 & 58 & 56 & 30 & 30 & 603 \\
\hline & $0.2 \%$ & $0.0 \%$ & $0.2 \%$ & $0.2 \%$ & $0.0 \%$ & $0.7 \%$ & $0.5 \%$ & $2.8 \%$ & $3.5 \%$ & $6.1 \%$ & $11.6 \%$ & $14.3 \%$ & $17.7 \%$ & $13.4 \%$ & $9.6 \%$ & $9.3 \%$ & $5.0 \%$ & $5.0 \%$ & $100.0 \%$ \\
\hline 2012 & 0 & 2 & 2 & 0 & 5 & 3 & 5 & 5 & 22 & 47 & 68 & 90 & 92 & 103 & 58 & 48 & 28 & 23 & 601 \\
\hline & $0.0 \%$ & $0.3 \%$ & $0.3 \%$ & $0.0 \%$ & $0.8 \%$ & $0.5 \%$ & $0.8 \%$ & $0.8 \%$ & $3.7 \%$ & $7.8 \%$ & $11.3 \%$ & $15.0 \%$ & $15.3 \%$ & $17.1 \%$ & $9.7 \%$ & $8.0 \%$ & $4.7 \%$ & $3.8 \%$ & $100.0 \%$ \\
\hline 2013 & 2 & 0 & 2 & 0 & 0 & 4 & 6 & 8 & 17 & 36 & 58 & 89 & 119 & 107 & 78 & 71 & 37 & 27 & 661 \\
\hline & $0.3 \%$ & $0.0 \%$ & $0.3 \%$ & $0.0 \%$ & $0.0 \%$ & $0.6 \%$ & $0.9 \%$ & $1.2 \%$ & $2.6 \%$ & $5.4 \%$ & $8.8 \%$ & $13.5 \%$ & $18.0 \%$ & $16.2 \%$ & $11.8 \%$ & $10.7 \%$ & $5.6 \%$ & $4.1 \%$ & $100.0 \%$ \\
\hline 2014 & 1 & 0 & 1 & 3 & 1 & 3 & 7 & 15 & 24 & 42 & 51 & 90 & 104 & 92 & 85 & 49 & 41 & 34 & 643 \\
\hline & $0.2 \%$ & $0.0 \%$ & $0.2 \%$ & $0.5 \%$ & $0.2 \%$ & $0.5 \%$ & $1.1 \%$ & $2.3 \%$ & $3.7 \%$ & $6.5 \%$ & $7.9 \%$ & $14.0 \%$ & $16.2 \%$ & $14.3 \%$ & $13.2 \%$ & $7.6 \%$ & $6.4 \%$ & $5.3 \%$ & $100.0 \%$ \\
\hline Total & 11 & 12 & 23 & 33 & 30 & 46 & 106 & 161 & 297 & 649 & 990 & 1375 & 1529 & 1441 & 1270 & 997 & 674 & 504 & 10148 \\
\hline
\end{tabular}

cancer (22.2\% alive at 5 years). Oral cavity, salivary gland, and laryngeal malignancies all saw mild improvements in 5 -year survival over the study period, while oropharyngeal and hypopharyngeal cancer survival improved significantly; $33.3 \%$ of oropharyngeal cancer patients survived to 5 years in 1994 compared to $55.1 \%$ in 2010 and $11.8 \%$ of hypopharyngeal cancer patients survived to 5 years compared to $36.7 \%$ in 2010.

\section{Discussion}

\section{Histology}

HNC is often considered analogous to SCC given the propensity for such lesions to develop in this region. This impacts significantly on management as a result of the relative radiosensitivity of SCCs as a group. From this 
Table 4 Overall and stratified by site statistics on disease stage at presentation

\begin{tabular}{|c|c|c|c|c|c|c|c|c|c|}
\hline Key & & & & & & & & & \\
\hline Frequ & & & & & & & & & \\
\hline Perce & age of Total & & & & & & & & \\
\hline Stage & Hypopharynx & Larynx & $\begin{array}{l}\text { Nasal Cavity I } \\
\text { Paranasal Sinuses }\end{array}$ & Nasopharynx & Oral Cavity & Oropharynx & Other & Salivary Gland & Total \\
\hline 0 & 0 & 4 & 0 & 0 & 0 & 0 & 1 & 0 & 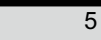 \\
\hline & 0 & 0.14 & 0 & 0 & 0 & 0 & 0.27 & 0 & 0.05 \\
\hline I & 15 & 753 & 3 & 8 & 791 & 110 & 39 & 142 & 1,861 \\
\hline & 1.89 & 26.44 & 0.62 & 2.62 & 26.12 & 6.85 & 10.66 & 19.72 & 18.34 \\
\hline II & 47 & 514 & 15 & 24 & 395 & 119 & 45 & 50 & 1,209 \\
\hline & 5.93 & 18.05 & 3.11 & 7.87 & 13.04 & 7.41 & 12.3 & 6.94 & 11.91 \\
\hline III & 76 & 384 & 21 & 32 & 308 & 215 & 38 & 28 & 1,102 \\
\hline & 9.58 & 13.48 & 4.36 & 10.49 & 10.17 & 13.39 & 10.38 & 3.89 & 10.86 \\
\hline IV & 228 & 480 & 50 & 36 & 741 & 697 & 117 & 120 & 2,469 \\
\hline & 28.75 & 16.85 & 10.37 & 11.8 & 24.47 & 43.4 & 31.97 & 16.67 & 24.33 \\
\hline IVC & 48 & 97 & 6 & 28 & 84 & 104 & 26 & 0 & 393 \\
\hline & 6.05 & 3.41 & 1.24 & 9.18 & 2.77 & 6.48 & 7.1 & 0 & 3.87 \\
\hline$x$ & 379 & 616 & 387 & 177 & 709 & 361 & 100 & 380 & 3,109 \\
\hline & 47.79 & 21.63 & 80.29 & 58.03 & 23.41 & 22.48 & 27.32 & 52.78 & 30.64 \\
\hline Total & 793 & 2,848 & 482 & 305 & 3,028 & 1,606 & 366 & 720 & 10,148 \\
\hline & 100 & 100 & 100 & 100 & 100 & 100 & 100 & 100 & 100 \\
\hline
\end{tabular}

cohort of 20 years of Irish patients, there was a lower proportion of SCC's than would have been expected. This category represents $84.2 \%$ of patients, a figure that published epidemiological data would indicate should exceed $95 \%$ [3, 7, 17-19]. It should be noted that the NCRI database included patients of all ages, including paediatric patients in whom a higher proportion of malignancies such as sarcoma and rarer malignancies not coded for would be more prevalent than SCC. In addition, a further $8.1 \%$ of the cases represented in this database were coded as unspecified carcinoma. This group may well be composed of SCC variants such as spindle cell, verrucous, and basaloid carcinomas as well as rarer diagnoses such as lymphoma and olfactory neuroblastoma $[20,21]$.

\section{Gender}

$74.15 \%$ of patients in this cohort were male, reflecting the selectivity HNC displays for male gender worldwide. It has been reported that the risk of HNC is twofold to fivefold higher in men than in women, with factors such as ethnicity, socioeconomic deprivation, and alcohol and smoking prevalence predicting the risk balance in any given population. The overall ratio in the United States of America (USA) has previously been reported as around three to one, which is reflected by the Irish data $[17,22]$.

\section{Age}

There was a broad age range represented in the reported data. While $\mathrm{HNC}$ is a rare diagnosis in the paediatric population, malignancy remains part of the differential for any mass in the head or neck. Previous data on paediatric HNC has indicated that where a single identifiable site can be identified, the most prevalent sites are the salivary glands, the nasopharynx, and the nasal cavity/middle ear [23, 24]. In this population, the latter of these findings were replicated, with patients under 20 presenting with nasopharyngeal, salivary gland, and nasal cavity/middle ear malignancies in $31 \%, 24 \%$, and $20 \%$ of cases, respectively. Of equal note is the overall distribution of cases- $48.15 \%$ of cases were in patients over the age of 65 , the prespecified definition of an 'elderly' patient for this study. Clearly, a significant proportion of patients with HNC are chronologically elderly at the time of diagnosis. Such patients were notably less likely to have formal documentation of disease stage - whether this is coincidental or due to much reduced life expectancy and perceived futility of treatment in the frail patient remains unclear.

\section{Incidence}

The incidence of HNC remained stable between 1994 and 2003. There was a steady increase in the number of HNC 


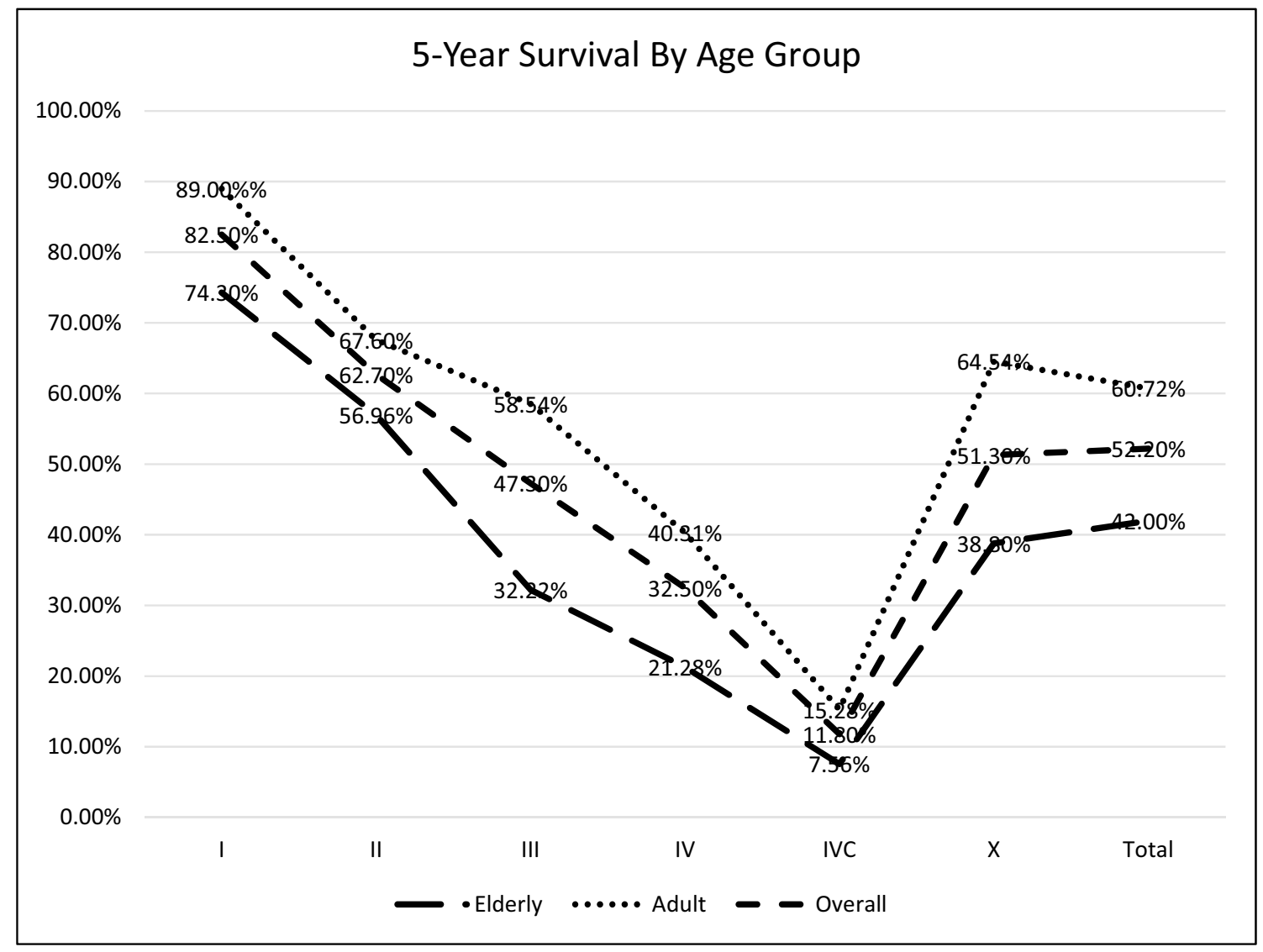

Fig. 1 Head and neck cancer 5-year survival by stage and age group

Table 5 -year survival by stage and year of incidence

\begin{tabular}{cccccc}
\hline YOI & Stage I & Stage II & Stage III & Stage IV & Total \\
\hline 1994 & $69.6 \%$ & $51.1 \%$ & $45.3 \%$ & $24.1 \%$ & $46.5 \%$ \\
1995 & $67.3 \%$ & $60.8 \%$ & $47.6 \%$ & $18.8 \%$ & $46.7 \%$ \\
1996 & $80.4 \%$ & $67.4 \%$ & $41.9 \%$ & $23.2 \%$ & $48.2 \%$ \\
1997 & $85.0 \%$ & $62.5 \%$ & $44.4 \%$ & $19.1 \%$ & $49.3 \%$ \\
1998 & $72.4 \%$ & $66.1 \%$ & $39.0 \%$ & $31.1 \%$ & $52.3 \%$ \\
1999 & $72.9 \%$ & $57.1 \%$ & $37.5 \%$ & $17.5 \%$ & $45.2 \%$ \\
2000 & $89.1 \%$ & $57.6 \%$ & $31.0 \%$ & $27.1 \%$ & $48.6 \%$ \\
2001 & $92.5 \%$ & $57.1 \%$ & $42.4 \%$ & $36.9 \%$ & $52.6 \%$ \\
2002 & $82.9 \%$ & $63.2 \%$ & $57.1 \%$ & $18.0 \%$ & $49.7 \%$ \\
2003 & $80.7 \%$ & $56.5 \%$ & $51.5 \%$ & $28.0 \%$ & $50.4 \%$ \\
2004 & $86.9 \%$ & $60.0 \%$ & $44.8 \%$ & $22.4 \%$ & $50.1 \%$ \\
2005 & $88.6 \%$ & $61.8 \%$ & $44.7 \%$ & $27.6 \%$ & $56.3 \%$ \\
2006 & $84.0 \%$ & $65.4 \%$ & $50.0 \%$ & $31.0 \%$ & $54.2 \%$ \\
2007 & $88.5 \%$ & $71.7 \%$ & $44.2 \%$ & $40.0 \%$ & $57.8 \%$ \\
2008 & $82.4 \%$ & $74.6 \%$ & $57.1 \%$ & $37.2 \%$ & $60.4 \%$ \\
2009 & $84.8 \%$ & $64.7 \%$ & $52.1 \%$ & $31.7 \%$ & $54.0 \%$ \\
2010 & $85.7 \%$ & $57.6 \%$ & $58.2 \%$ & $38.4 \%$ & $56.8 \%$ \\
\hline & & & & &
\end{tabular}

cases diagnosed each year in Ireland between 2004 and 2014, from 452 to 643 . Worldwide, there has been a similar trend in HNC diagnoses, but the face of this entity is changing, with the incidence of malignancies at certain sites (such as laryngeal and nasopharyngeal) decreasing in frequency as a direct result of public health campaigns to reduce tobacco and alcohol consumption. There has been a concurrent rise in the incidence of oropharyngeal and hypopharyngeal malignancy due in no small part to the rising prevalence of HPV infection [25]. In the reported data there was no notable effect on the incidence of laryngeal or nasopharyngeal cancers before, during, or after the 2004 smoking ban came into effect in Ireland, though a small decrease in oral cavity cancer was noted. There has, however, been a gradual increase in the prevalence of oropharyngeal malignancies, from $13.59 \%$ in 1994 to $22.24 \%$ in 2014. HPV positive cancers are more common in white, socioeconomically privileged populations - this trend is unsurprising given the predominance of white ethnicity in Ireland and the relatively high socioeconomic status of the country as a whole [26].

The data presented in Table 3 also reflects the growing prevalence of HPV driven malignancies-the growing prevalence of young HNC patients has been well-described, and 
Table 6 5-year survival by site and year of incidence

\begin{tabular}{|c|c|c|c|c|c|c|c|c|}
\hline YOI & Hypopharynx & Larynx & $\begin{array}{l}\text { Nasal cavity/para- } \\
\text { nasal sinuses }\end{array}$ & Nasopharynx & Oral cavity & Oropharynx & Salivary gland & Total \\
\hline 1994 & $11.8 \%$ & $55.3 \%$ & $58.3 \%$ & $42.9 \%$ & $54.9 \%$ & $33.3 \%$ & $56.0 \%$ & $46.5 \%$ \\
\hline 1995 & $17.9 \%$ & $55.2 \%$ & $50.0 \%$ & $58.3 \%$ & $52.3 \%$ & $33.3 \%$ & $62.5 \%$ & $46.7 \%$ \\
\hline 1996 & $22.0 \%$ & $59.3 \%$ & $15.4 \%$ & $30.8 \%$ & $60.9 \%$ & $34.2 \%$ & $60.0 \%$ & $48.2 \%$ \\
\hline 1997 & $15.2 \%$ & $69.6 \%$ & $52.6 \%$ & $53.9 \%$ & $55.2 \%$ & $32.0 \%$ & $46.7 \%$ & $49.3 \%$ \\
\hline 1998 & $33.3 \%$ & $57.1 \%$ & $50.0 \%$ & $50.0 \%$ & $57.3 \%$ & $41.7 \%$ & $62.1 \%$ & $52.3 \%$ \\
\hline 1999 & $23.1 \%$ & $54.3 \%$ & $53.9 \%$ & $46.7 \%$ & $53.3 \%$ & $31.7 \%$ & $38.1 \%$ & $45.2 \%$ \\
\hline 2000 & $8.8 \%$ & $61.1 \%$ & $41.2 \%$ & $70.0 \%$ & $54.6 \%$ & $34.0 \%$ & $62.5 \%$ & $48.6 \%$ \\
\hline 2001 & $27.3 \%$ & $55.1 \%$ & $53.9 \%$ & $38.5 \%$ & $63.5 \%$ & $48.7 \%$ & $40.0 \%$ & $52.6 \%$ \\
\hline 2002 & $19.1 \%$ & $52.5 \%$ & $60.0 \%$ & $50.0 \%$ & $52.6 \%$ & $43.1 \%$ & $61.1 \%$ & $49.7 \%$ \\
\hline 2003 & $32.3 \%$ & $53.4 \%$ & $70.6 \%$ & $75.0 \%$ & $56.1 \%$ & $37.3 \%$ & $48.2 \%$ & $50.4 \%$ \\
\hline 2004 & $15.2 \%$ & $60.8 \%$ & $45.0 \%$ & $69.2 \%$ & $52.6 \%$ & $43.8 \%$ & $41.7 \%$ & $50.1 \%$ \\
\hline 2005 & $21.2 \%$ & $59.7 \%$ & $50.0 \%$ & $36.4 \%$ & $65.3 \%$ & $56.9 \%$ & $67.9 \%$ & $56.3 \%$ \\
\hline 2006 & $31.6 \%$ & $52.9 \%$ & $53.3 \%$ & $38.5 \%$ & $63.5 \%$ & $52.6 \%$ & $70.4 \%$ & $54.2 \%$ \\
\hline 2007 & $14.3 \%$ & $63.1 \%$ & $61.1 \%$ & $53.9 \%$ & $67.2 \%$ & $56.9 \%$ & $70.0 \%$ & $57.8 \%$ \\
\hline 2008 & $25.0 \%$ & $63.8 \%$ & $71.4 \%$ & $50.0 \%$ & $69.4 \%$ & $48.4 \%$ & $66.7 \%$ & $60.4 \%$ \\
\hline 2009 & $27.1 \%$ & $62.2 \%$ & $46.7 \%$ & $69.2 \%$ & $56.5 \%$ & $53.1 \%$ & $58.1 \%$ & $54.0 \%$ \\
\hline 2010 & $36.7 \%$ & $58.7 \%$ & $75.0 \%$ & $56.5 \%$ & $61.5 \%$ & $55.1 \%$ & $71.0 \%$ & $56.8 \%$ \\
\hline Total & $22.2 \%$ & $58.6 \%$ & $54.3 \%$ & $52.6 \%$ & $58.9 \%$ & $44.8 \%$ & $58.6 \%$ & $51.2 \%$ \\
\hline
\end{tabular}

it has been shown that most HPC driven malignancies present at a younger age than their non HPV associated counterparts [27]. Equally worthy of note is the growing population of HNC patients over 85 years of age, a group whose numbers doubled over the 20 years of the study period.

\section{Stages and survival}

The two chief components of the poor prognosis associated with HNC in general are the often advanced nature of the disease at presentation and the potential it possesses for locoregional or metastatic recurrence. It has previously been reported that up to $70 \%$ of HNC possesses some element of advanced disease at presentation [14]. 39.1\% of patients presented with documented locoregionally advanced or metastatic disease in Ireland in the given period, but this number is likely an underestimation- $30.6 \%$ of cases did not have documentation of their staging. While 643 of the 3109 cases represented here came about as a result of 2014 cases having no record of staging data, even when these are excluded, stage $\mathrm{X}$ disease still comprised $25.9 \%$ of the dataset. On further interrogation of this group, it contained a higher proportion of older patients than any other stage group. In addition, Table 4 demonstrates that patients with nasal cavity and paranasal sinus malignancies were overwhelmingly more likely than any other site not to have documented staging status, though salivary gland and nasopharyngeal malignancy were also overrepresented in this regard. It remains challenging to draw any definitive conclusions on this group, but it can be assumed that the predominant component of the group is advanced disease given the survival statistics in Fig. 1 place it somewhere closest to stage III in terms of prognosis. When patients who lacked formally documented staging were excluded, the proportion of patients who presented with locoregionally advanced or metastatic disease was $56.3 \%$.

The overall 5-year cancer survival for HNC observed in the Irish population was $52.2 \%$, though significant variability was observed when site and stage at presentation are considered. This is in line with HNC survival statistics for other European countries, and above the reported European average of $39.9 \%$, though it should borne in mind that this figure includes data Eastern European where the survival was significantly lower than other regions [28, 29]. Stage I and II disease were associated with relatively high 5-year survival at $82.5 \% \%$ and $62.7 \%$, respectively. Stage IVC disease was associated with a dismal 5-year survival of $11.8 \%$. Laryngeal cancer 5-year survival at $58.6 \%$ is on par with the European average of $58.9 \%$ while oral cavity 5 year-survival at $58.9 \%$ is well above the average of $45.4 \%$, though this has been noted to be an area significantly contributed to by the overwhelming preponderance of such cases in Eastern Europe. Overall the best survival was associated with oral cavity, laryngeal, and salivary gland cancers, which is consistent with internationally reported survival statistics [8, 22, 30, 31].

Perhaps most noteworthy is the improvement in survival which has been observed in this time frame, most notable 
in the oropharynx and hypopharynx but also observed in the oral cavity, salivary glands, and larynx. This is at odds with much published international data, including a relatively recent paper from 2010 by Guntinas-Lichius et al. [32] who reported quite similar data on a large cohort of German HNC patients. Despite similar baseline characteristics, survival in this cohort was slightly lower overall and particularly in the oral cavity, larynx, and nasopharynx. Also described were similar data handling issues; specifically noted were a percentage of cases that lacked formally designated site data, though the prevalence of this was lower than the data presented in this study. More precise comparison of the differences in survival would be aided by more detailed staging data — as highlighted above the Irish data most likely underestimates the number of advanced cases owing to the number of stage $X$ cases.

\section{Conclusion}

There is a growing population of young HNC patients in Ireland driven largely by the growing prevalence of HPV associated malignancies. Survival is improving in Ireland but remains poor overall due to the often advanced nature of disease at presentation. The elderly HNC patient continues to pose challenges that are best resolved by close adherence to multidisciplinary assessment, development and encouragement of subspecialisation in cancer care, and use of targeted therapies based on tumour subsites.

Author contributions Concept and design: GPS, JPO. Acquisition, analysis, or interpretation of data: GPS, PW, JPO. Drafting of the manuscript: GPS. Critical revision of the manuscript for important intellectual content: GPS, FM, JPO. Statistical analysis: GPS, FM

Funding Open Access funding provided by the IReL Consortium.

\section{Declarations}

Conflict of interest We here declare that we have no conflicting/competing interests.

Availability of data and material Stored and maintained by National Cancer Registry Ireland.

Code availability Stata 16.1

Ethical approval Approval was sought and received from the RCSI Research Ethics Committee.

Open Access This article is licensed under a Creative Commons Attribution 4.0 International License, which permits use, sharing, adaptation, distribution and reproduction in any medium or format, as long as you give appropriate credit to the original author(s) and the source, provide a link to the Creative Commons licence, and indicate if changes were made. The images or other third party material in this article are included in the article's Creative Commons licence, unless indicated otherwise in a credit line to the material. If material is not included in the article's Creative Commons licence and your intended use is not permitted by statutory regulation or exceeds the permitted use, you will need to obtain permission directly from the copyright holder. To view a copy of this licence, visit http://creativecommons.org/licenses/by/4.0/.

\section{References}

1. Huang SH, O'Sullivan B (2017) Overview of the 8th editionTNM classification for head and neck cancer. Curr Treat Options Oncol 18(7):40

2. Yancik R, Ries LA (2000) Aging and cancer in America. Demographic and epidemiologic perspectives. Hematol Oncol Clin $\mathrm{N}$ Am 14(1):17-23

3. Mifsud M, Eskander A, Irish J, Gullane P, Gilbert R, Brown D et al (2017) Evolving trends in head and neck cancer epidemiology: Ontario, Canada 1993-2010. Head Neck 39(9):1770-1778

4. Porceddu SV, Haddad RI (2017) Management of elderly patients with locoregionally confined head and neck cancer. Lancet Oncol 18(5):e274-e283

5. Johnson S, McDonald JT, Corsten MJ (2008) Socioeconomic factors in head and neck cancer. J Otolaryngol Head Neck Surg 37(4):597-601

6. (2017) International head and neck cancer epidemiology consortium: update No. 23. Head Neck 39(7):1479

7. Mourad M, Jetmore T, Jategaonkar AA, Moubayed S, Moshier E, Urken ML (2017) Epidemiological trends of head and neck cancer in the United States: a SEER population study. J Oral Maxillofac Surg 75(12):2562-2572

8. Du E, Mazul AL, Farquhar D, Brennan P, Anantharaman D, Abedi-Ardekani B et al (2019) Long-term survival in head and neck cancer: impact of site, stage, smoking, and human papillomavirus status. Laryngoscope 129(11):2506-2513

9. Kobayashi K, Hisamatsu K, Suzui N, Hara A, Tomita H, Miyazaki T (2018) A review of HPV-related head and neck cancer. J Clin Med 7(9):241

10. Heffernan CB, O'Neill JP, Timon C (2010) Oncogenic impact of human papilloma virus in head and neck cancer. J Laryngol Otol 124(9):941-944

11. Kreimer AR, Clifford GM, Boyle P, Franceschi S (2005) Human papillomavirus types in head and neck squamous cell carcinomas worldwide: a systematic review. Cancer Epidemiol Biomark Prev 14(2):467

12. Tsao SW, Tsang CM, Lo KW (2017) Epstein-Barr virus infection and nasopharyngeal carcinoma. Philos Trans R Soc Lond B Biol Sci 372(1732):20160270

13. Raghupathy R, Hui EP, Chan AT (2014) Epstein-Barr virus as a paradigm in nasopharyngeal cancer: from lab to clinic. Am Soc Clin Oncol Educ Book 34:149-153

14. Guizard A-VN, Dejardin OJ, Launay LC, Bara S, Lapôtre-Ledoux BM, Babin EB, et al. Diagnosis and management of head and neck cancers in a high-incidence area in France: a population-based study. Medicine (Baltimore). 2017;96(26):e7285-e.

15. The National Cancer Registry Board (Establishment) Order, 1991, Stat. 19/1991 (1991).

16. Amin M ES, Greene F, et al. AJCC cancer staging manual. Eighth Edition ed. New York: Springer; 2017.

17. Rettig EM, D'Souza G (2015) Epidemiology of head and neck cancer. Surg Oncol Clin 24(3):379-396

18. Cohen N, Fedewa S, Chen AY (2018) Epidemiology and demographics of the head and neck cancer population. Oral Maxillofac Surg Clin North Am 30(4):381-395

19. Bray F, Ferlay J, Soerjomataram I, Siegel RL, Torre LA, Jemal A (2018) Global cancer statistics 2018: GLOBOCAN estimates of 
incidence and mortality worldwide for 36 cancers in 185 countries. CA Cancer J Clin 68(6):394-424

20. Helliwell TR, Giles TE (2016) Pathological aspects of the assessment of head and neck cancers: United Kingdom National Multidisciplinary Guidelines. J Laryngol Otol 130(S2):S59-S65

21. Pai SI, Westra WH (2009) Molecular pathology of head and neck cancer: implications for diagnosis, prognosis, and treatment. Annu Rev Pathol 4:49-70

22. Howlader N NA, Krapcho M, Miller D, Brest A, Yu M, Ruhl J, Tatalovich Z, Mariotto A, Lewis DR, Chen HS, Feuer EJ, Cronin KA (eds). SEER Cancer Statistics Review, 1975-2017. National Cancer Institute. Bethesda, MD; 2020.

23. Sengupta S, Pal R, Saha S, Bera SP, Pal I, Tuli IP (2009) Spectrum of head and neck cancer in children. J Indian Assoc Pediatr Surg 14(4):200-203

24. Cesmebasi A, Gabriel A, Niku D, Bukala K, Donnelly J, Fields PJ et al (2014) Pediatric head and neck tumors: an intra-demographic analysis using the SEER* database. Med Sci Monit 20:2536-2542

25. Aupérin A (2020) Epidemiology of head and neck cancers: an update. Curr Opin Oncol 32(3):178-186

26. Office CS. Press Statement Census 2016 Results Profile 8 - Irish Travellers, Ethnicity and Religion. 2016.

27. Van Dyne EA, Henley SJ, Saraiya M, Thomas CC, Markowitz LE, Benard VB (2018) Trends in human papillomavirus-associated cancers-United States, 1999-2015. MMWR Morb Mortal Wkly Rep 67(33):918-924
28. De Angelis R, Sant M, Coleman MP, Francisci S, Baili P, Pierannunzio D et al (2014) Cancer survival in Europe 1999-2007 by country and age: results of EUROCARE-5-a population-based study. Lancet Oncol 15(1):23-34

29. Gatta G, Botta L, Sánchez MJ, Anderson LA, Pierannunzio D, Licitra L et al (2015) Prognoses and improvement for head and neck cancers diagnosed in Europe in early 2000s: The EUROCARE-5 population-based study. Eur J Cancer 51(15):2130-2143

30. Cadoni G, Giraldi L, Petrelli L, Pandolfini M, Giuliani M, Paludetti G et al (2017) Prognostic factors in head and neck cancer: a 10 -year retrospective analysis in a single-institution in Italy. Acta Otorhinolaryngol Ital 37(6):458-466

31. Pulte D, Brenner H (2010) Changes in survival in head and neck cancers in the late 20th and early 21 st century: a period analysis. Oncologist 15(9):994-1001

32. Guntinas-Lichius O, Wendt T, Buentzel J, Esser D, Lochner P, Mueller A et al (2010) Head and neck cancer in Germany: a sitespecific analysis of survival of the Thuringian cancer registration database. J Cancer Res Clin Oncol 136(1):55-63

Publisher's Note Springer Nature remains neutral with regard to jurisdictional claims in published maps and institutional affiliations. 